\title{
PEDIATRIC ORIGINAL ARTICLE Identification of a dietary pattern prospectively associated with increased adiposity during childhood and adolescence
}

\author{
GL Ambrosini ${ }^{1}$, PM Emmett ${ }^{2}, \mathrm{~K}_{\text {Northstone }}{ }^{2}$, LD Howe $^{2}, \mathrm{~K}$ Tilling $^{2}$ and SA Jebb ${ }^{1}$
}

BACKGROUND: Specific dietary risk factors for excess adiposity in young people are poorly understood. However, studies in adults suggest dietary energy density, fat and fibre are critical dietary factors.

OBJECTIVE: To examine longitudinal relationships between a dietary pattern (DP) characterised by dietary energy density, \% total energy from fat and fibre density and fat mass (FM) in children from 7 to 15 years of age.

DESIGN: Subjects were 6772 children from the UK Avon Longitudinal Study of Parents and Children. Dietary intake was assessed using a 3-day food diary at 7, 10 and 13 years of age. An energy-dense, high-fat, low-fibre DP was identified using reduced rank regression and subjects scored for the DP at each age. FM was measured at 11, 13 and 15 years and FM index (FMI) calculated as FM/height ${ }^{(x)}$. Longitudinal models were adjusted for dietary misreporting, physical activity and maternal factors.

RESULTS: DP $z$-scores at all ages were positively associated with later FMI. A 1 s.d. unit increase in DP z-score was longitudinally associated with an average increase in FMI $z$-score of 0.04 s.d. units ( $95 \%$ confidence interval $(\mathrm{Cl}), 0.01-0.07)$. For each 1 s.d. unit increase in DP $z$-score, the odds of being in the highest quintile for FMI (as a marker of excess adiposity) increased by $13 \%$ (95\% Cl, 1-27\%).

CONCLUSIONS: Dietary habits during childhood are associated with increased adiposity in adolescence, with specific implications for dietary energy density, fat and fibre intake. Improving diet quality may reduce the risk of obesity in young people.

International Journal of Obesity (2012) 36, 1299-1305; doi:10.1038/ijo.2012.127; published online 7 August 2012

Keywords: diet; dietary patterns; fat mass; adolescents; children; ALSPAC

\section{INTRODUCTION}

The prevalence of childhood overweight and obesity is a major public health concern. Dietary intake is an important determinant of energy balance, but it is difficult to accurately measure energy intake (El) in large population studies. Estimated El is prone to dietary underreporting regardless of the dietary assessment method, but this is often overlooked. The resulting imprecision in El measurement makes it difficult to identify associations between El and weight gain.

By comparison, dietary composition may be more readily characterised and could influence obesity risk via its indirect effects on El. Experimental research provides clear evidence that energy-dense diets undermine innate appetite control and are associated with greater energy consumption. ${ }^{1}$ It has been demonstrated that innate appetite control is strongest in young children and diminishes in older children. ${ }^{2}$ This, in combination with under-developed cognitive dietary restraint mechanisms, may make older children more susceptible to consuming energydense diets. ${ }^{3}$ Energy-dense diets are typically high in fat and low in fibre. Dietary fat contributes more energy per unit weight and is metabolised at lower energetic cost than other macronutrients. ${ }^{4}$ Fat is also less satiating relative to other macronutrients, although this may be modified by the energy density of high-fat foods. ${ }^{5}$ Evidence supporting dietary fat in the development of obesity beyond its contribution to $\mathrm{El}$ is inconclusive ${ }^{6}$ although intervention studies demonstrate that reductions in fat intake can result in weight loss. ${ }^{7}$ In contrast, high-fibre diets tend to be low in energy density and are associated with lower obesity risk. ${ }^{8}$
Dietary fibre increases satiety levels ${ }^{8}$ and this may delay or decrease subsequent El. In 2003 the WHO identified energy-dense diets and low fibre intake as important determinants of obesity, ${ }^{8}$ however, most of the evidence was based on studies in adults. Surprisingly few longitudinal studies have examined diet and obesity risk in children or adolescents.

To date, much of the published research on diet and obesity has focussed on single foods or nutrients. In recent decades, dietary pattern (DP) analyses have been increasingly used to consider total food intake and the potentially synergistic effects of foods and nutrients. Empirical DPs take advantage of the colinearity between dietary factors to examine their collective influence, whereas attempting to separate individual nutrient's effects may be inappropriate or their impact too small to detect. ${ }^{9}$

Few studies have examined DPs in relation to obesity in children or adolescents. Of these, most have applied exploratory methods such as principal components analysis or factor analysis to identify DPs. An emerging statistical method, reduced rank regression (RRR) incorporates a priori knowledge into exploratory analyses to identify DPs so that specific hypothesised pathways between dietary intake and health outcomes may be tested.

We previously identified a DP high in energy density and fat and low in fibre, using the RRR method in a subgroup from a large UK birth cohort, the Avon Longitudinal Study of Parents and Children (ALSPAC). ${ }^{10}$ In an analysis of 600 children, higher scores for this DP at 5 and 7 years of age were associated with a greater fat mass (FM) and increased risk of excess adiposity at 9 years. ${ }^{10}$ The current paper extends this work by analysing follow-up to

${ }^{1}$ Medical Research Council (MRC) Human Nutrition Research, Cambridge, UK and ${ }^{2}$ School of Social and Community Medicine, University of Bristol, Bristol, UK. Correspondence: Dr GL Ambrosini, Medical Research Council (MRC) Human Nutrition Research, 120 Fulbourn Road, Cambridge CB1 9NL, UK.

E-mail: Gina.Ambrosini@mrc-hnr.cam.ac.uk

Received 28 February 2012; revised 9 May 2012; accepted 11 July 2012; published online 7 August 2012 
15 years in the full ALSPAC cohort of over 6500 boys and girls. We hypothesised that the same energy-dense, high-fat, low-fibre DP would be observed at 7, 10 and 13 years of age, and that this pattern would be prospectively associated with greater body fatness at 11, 13 and 15 years of age. As innate appetite control is stronger at younger ages, a second hypothesis was that the relationship between this DP and body fatness would strengthen with age.

\section{SUBJECTS AND METHODS}

Full details of ALSPAC have been reported elsewhere. ${ }^{11}$ In brief, ALSPAC recruited 14541 pregnant women residing in Avon, England with an expected delivery date between 1 April 1991 and 31 December 1992. A known birth outcome was available for 14472 pregnancies resulting in 14062 live births of which, 13988 were alive at 1 year. At the 7 year follow-up an additional 548 children were recruited who were initially invited to the study but declined. Thus, the total baseline cohort is 14536 children alive at 1 year. A range of data has been collected from the women, their partners and their children, antenatally and at regular intervals thereafter, via questionnaires and clinic visits. The number attending clinic visits at the 7,10,11,13 and 15 years of age was 8297, $7563,7159,6147$ and 5509, respectively.

\section{Dietary assessments}

A 3-day unweighed food diary was sent to child participants before clinic visits for the 7, 10 and 13 years follow-ups. Parents completed the diaries at 7 years of age, the children completed the food diary at 10 and 13 year with parental input. Respondents were requested to complete the diary over 2 weekdays and 1 weekend day (non-consecutive) and these were checked and queried with the child and parent by a nutritionist at clinic visits. The food diaries were coded and linked to food composition tables using DIDO, a programme developed at MRC Human Nutrition Research (Cambridge). Nutrient intakes were calculated using McCance and Widdowson's British food composition tables. Intakes of individual foods were combined into predefined Cambridge Food Groups used in previous analyses of ALSPAC dietary data. ${ }^{10}$

An individualised method was used to identify dietary misreporting based on the ratio of El to estimated energy requirement (EER). ${ }^{12,13}$ To take account of the variation in the methods used to estimate El and EER, a $95 \%$ confidence interval (Cl) was calculated for El:EER. ${ }^{14}$ Individuals were classified as plausible reporters (El:EER within the $95 \% \mathrm{Cl}$ for El:EER), overreporters (El:EER $>95 \% \mathrm{Cl}$ for El:EER) or underreporters (El:EER $<95 \% \mathrm{Cl}$ El:EER) and this was included as categorical covariate in all analyses.

\section{Dietary patterns}

RRR was applied to identify a DP characterised by dietary energy density, \% energy from fat and dietary fibre density. RRR identifies patterns in a set of predictor variables that explain the maximum variation in another set of variables known as response variables, which are hypothesised to be intermediates between the predictor variables and a health outcome of interest. $^{15}$

Using PROC PLS in SAS ${ }^{16}$ the RRR model included all food group intakes (g per day) as predictor variables and dietary energy density, \% energy from fat and fibre density as response variables. Dietary energy density $\left(\mathrm{KJ} \mathrm{g}^{-1}\right)$ was calculated as total food energy $(\mathrm{kJ})$ divided by total food weight $(\mathrm{g})$, excluding beverages. ${ }^{17}$ Dietary fibre density $\left(\mathrm{g} \mathrm{MJ}^{-1}\right)$ was calculated as total $\mathrm{g}$ fibre (nonstarch polysaccharide) divided by total EI (MJ). The \% energy from fat was calculated as $\mathrm{g}$ total fat multiplied by $37 \mathrm{~kJ}$ then divided by total El ( $\mathrm{JJ}$ ), and multiplied by 100 . Separate RRR analyses were conducted using dietary data from 7, 10 and 13 years of age, and by gender. As there were no major gender differences at any age, reported DPs are based on boys and girls combined.

The first DP from each RRR analyses explained the greatest variation in all three response variables (45\%, all ages) compared with the second and third patterns, which explained $\sim 15 \%$ and $9 \%$, respectively (all ages). Therefore, only the first pattern DP was considered for further analyses. This DP was positively associated with dietary energy density and \% energy from fat, and negatively associated with dietary fibre density (Supplementary Table 1) and labelled an 'energy-dense, high-fat, low-fibre dietary pattern'. This pattern explained $\sim 64 \%, 51 \%$ and $20 \%$ of the variation in dietary energy density, fibre density and \% energy from fat, respectively (Supplementary Table 1).

Factor loadings for the energy-dense, high-fat, low-fibre DP were consistent at 7, 10 and 13 years of age (Figure 1). At all ages, the DP was most strongly characterised by low intakes of fruit and vegetables (Figure 1). At 7 years of age, over $80 \%$ of the variation in DP scores was explained by the top five and bottom five factor loadings, with fresh fruit explaining the most variation (27\%), then vegetables (raw or cooked, $16 \%)$, legumes $(7 \%)$, high-fibre breakfast cereal $(6 \%)$, confectionery and chocolate (5\%). This was similar at 10 and 13 years of age. These characteristics are almost identical to the energy-dense, high-fat, low-fibre DP identified in a subset of this cohort at 5 and 7 years of age. ${ }^{10}$

Each respondent received a $z$-score quantifying the degree to which their reported dietary intake reflected this DP at 7, 10 and 13 years. Although a very similar energy-dense, high-fat, low-fibre DP was observed in each RRR analyses, a score for exactly the same DP (based on the same covariance matrix) at 7, 10 and 13 years was required to assess longitudinal changes in $z$-scores for the same DP in relation to FM. Using confirmatory $\mathrm{RRR}^{18}$ applied DP scores were calculated at 10 and 13 years of age using scoring weights from the energy-dense, high-fat, low-fibre DP identified at 7 years.

\section{Adiposity}

FM was measured by dual-energy X-ray absorptiometry (DXA) at the 11-, 13- and 15-year follow-ups using the Lunar Prodigy DXA fan bean scanner (GE Medical Systems Lunar, Madison, WI, USA). FM index (FMI) was calculated as FM $(\mathrm{kg})$ divided by height $(\mathrm{m})$ raised to an optimum power $(x)$ to remove the relation between $\mathrm{FMl}$ and height, that is, FM/height ${ }^{x} .^{19}$ The optimum power $(x)$ was calculated by regressing log (FM) on log (height). ${ }^{19}$ As the optimum power $(x)$ varied significantly by gender and age (boys: 5.3 at 11 years, 1.8 at 13 years, 2.4 at 15 years; girls: 4.2 at 11 years, 3.1 at 13 years and 2.4 at 15 years), FMI was calculated separately for boys and girls and at each follow-up. To allow same scale comparisons across all ages, FMI was log-transformed to obtain normal distributions and standardised to a $z$-score. Individuals in the top quintile (above 80th percentile) of FMI $z$-scores were classified as having excess adiposity.

\section{Other variables}

Height was measured at each follow-up to the nearest $0.1 \mathrm{~cm}$, without shoes and socks, using a Harpenden stadiometer (Holtain Ltd, Crymmych, Pembs, UK). Weight was measured to the nearest $0.1 \mathrm{~g}$ with Tanita body fat analyser weighing scales (Tanita, West Drayton, Middlesex, UK). Pubertal status was self-reported at 11 and 13 years using diagrams depicting the five Tanner Stages ${ }^{20}$ for pubic hair development as this has been reported as reliable for assessing maturation in this cohort. ${ }^{21}$ Total physical activity was measured at the 11 and 13 years using an MTI Actigraph AM7164 2.2 accelerometer (Actigraph LLC, Fort Walton Beach, FL, USA) worn for at least $10 \mathrm{~h}$ per day for 3 days. ${ }^{22}$ Total physical activity was measured as counts $\min ^{-1}(\mathrm{cpm})$ averaged over the number of valid days recorded (minimum of 3 days) and log-transformed to achieve a normal distribution. Maternal education level and maternal prepregnancy body mass index (BMI) was assessed by questionnaire at 32 week's gestation.

\section{Ethics}

Parents provided written consent for their child to participate in the study. Ethical approval for the study was obtained from the ALSPAC Law and Ethics Committee and the Local Research Ethics Committees.

\section{Statistical analyses}

Descriptive analyses compared child characteristics and selected dietary intakes according to quintiles of the DP score. Analysis of variance was used to estimate mean values and trends across quintiles of the DP score adjusting for sex and age. Trends in the proportions overweight or obese (BMI) or with excess adiposity (FMI) were compared across quintiles of the DP score using a two-sided Cochrane-Armitage test.

Generalised linear models were applied to examine prospective associations between DP $z$-scores at 7, 10 and 13 years of age and FM at 11,13 and 15 years of age, respectively. All models were adjusted for dietary misreporting and exact age at the time of dietary assessment. As no interactions were observed between the DP and gender, data from boys and girls were analysed together and all models were adjusted for gender. Additional models were adjusted for physical activity and Tanner 


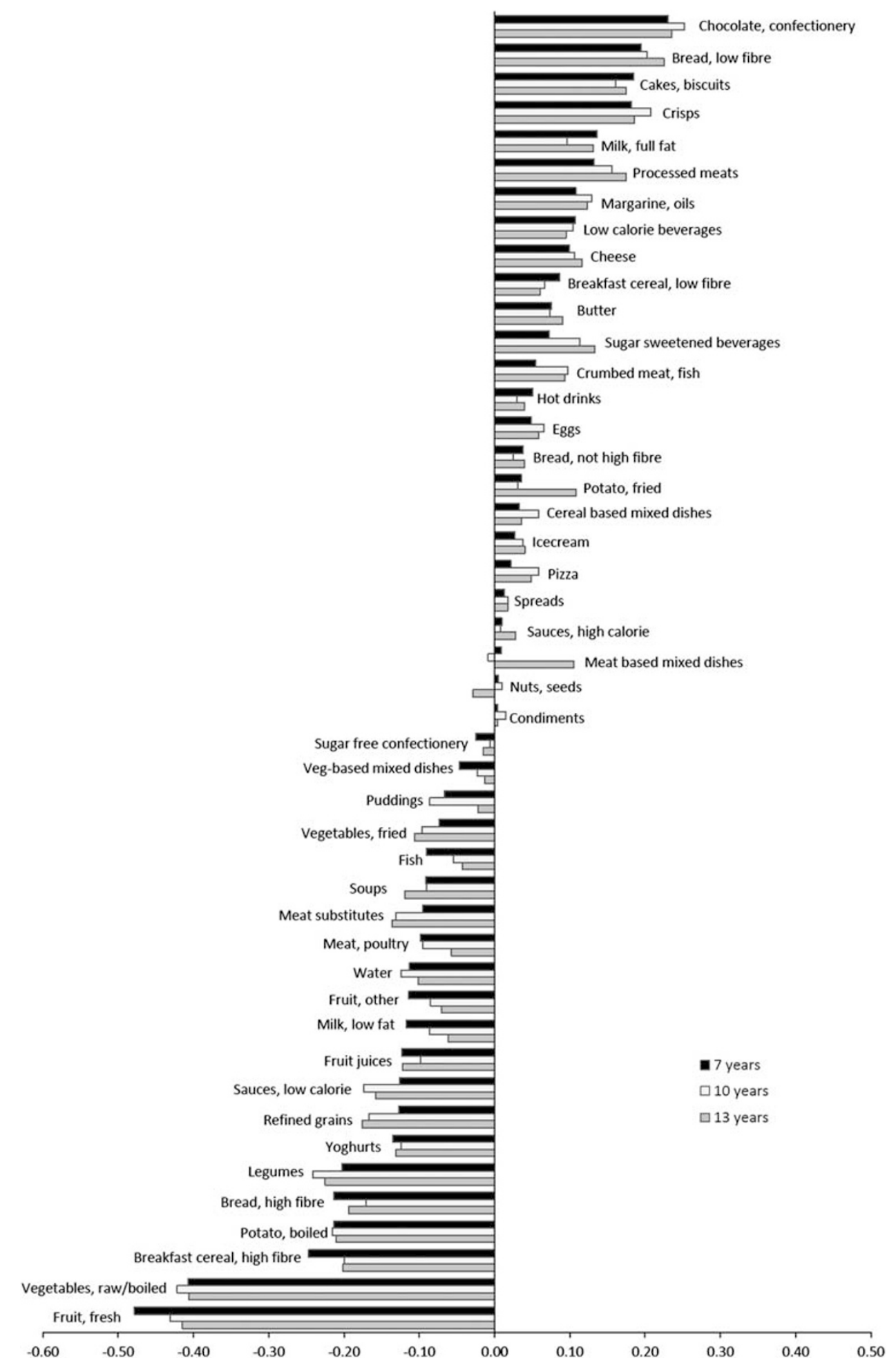

Figure 1. Factor loadings for the energy-dense, high-fat, low-fibre DP at 7, 10 and 13 years of age in the ALSPAC cohort.

stage. Maternal education and prepregnancy BMI have been associated with obesity in the ALSPAC cohort ${ }^{23}$ and were tested in separate models. All generalised linear models were run using PROC REG in SAS. ${ }^{16}$

Generalised estimating equation (GEE) models were applied to assess whether the association between DP and FMI $z$-scores changed with age. These longitudinal models regressed FMI on DP score at the previous time point, using DP $z$-scores from 7 to 13 years of age and FMI $z$-scores from 11 to 15 years of age. A GEE model was used to examine the odds of excess adiposity in relation to DP $z$-score. GEE models were run using the xtgee command in Stata with an exchangeable correlation structure. ${ }^{24}$ All models included exact age at dietary assessment, exact age at outcome and dietary misreporting as time-varying covariates. Additional models also adjusted for physical activity and tanner stage as time-varying covariates, and maternal education and prepregnancy BMI as fixed variables. As there was no interaction between gender and DP scores, boys and girls were analysed together, adjusting for gender. To test if the strength of association (if any) between the DP and FMl varied by age, an interaction term between DP $z$-score and age of dietary assessment was tested in each model. The DP $z$-scores were also analysed in quintiles, to test if associations were linear. 


\section{RESULTS}

Mean height and FM at each follow-up showed differences between boys and girls expected with pubertal development (Table 1). With increasing age, average total FM in girls exceeded that of boys, whereas the average height of boys exceeded girls. Girls had a higher prevalence of overweight or obesity based on $\mathrm{BMI}$, at all ages.

Food diary data were available for 7285,7471 and 6106 children at 7, 10 and 13 years of age, respectively. Seventy three per cent, $63 \%$ and $39 \%$ of respondents were defined as plausible reporters at 7, 10 and 13 years, respectively. An increasing score for the energy-dense, high-fat, low-fibre DP at 7 years of age was associated with greater intakes of dietary energy density, $\%$ energy from fat and saturated fat, and lower fibre density, \% energy from protein and carbohydrate, vitamin C and folate (Supplementary Table 2). Associations were similar at 10 and 13 years of age (data not shown).

There was an increasing trend in mean FMI and the proportion of subjects with excess adiposity at 11 years of age across quintiles of the DP score at 7 years ( $P$-value for trend $<0.001$, Table 2 ). A weaker trend was apparent between DP scores at 10 years and FMI measured at 13 years of age. The proportion of subjects classified as overweight or obese according to BMI at 11 years of age increased across quintiles of the DP measured at 7 years $(P$-value for trend $<0.0001$, Table 2$)$. There were no statistical or clinical differences in mean BMI across quintiles of the DP scores at any age.

Multiple linear regression models showed that at all ages, the energy-dense, high-fat, low-fibre DP was positively associated with subsequent FMI. A 1 s.d. greater $z$-score for the DP at 7 years of age was associated with a FMI $z$-score at 11 years of age that was greater on average, by 0.10 s.d. units $(95 \% \mathrm{Cl}, 0.07-0.12)$ (Model 1, Table 3). A similar sized association was observed between the DP measured at 7 years and FMI $z$-score at 13 years $(0.09,95 \% \mathrm{Cl}, 0.06-0.11)$ and 15 years of age $(0.09,95 \% \mathrm{Cl}$, $0.06-0.12)$. DP scores at 10 years of age showed similar associations with later FMI. The weakest association was observed between the DP $z$-score at 13 years of age and FMl at 15 years $(0.03,95 \% \mathrm{Cl}, 0.01-0.05)$. Adjusting for physical activity had no appreciable effect on these associations (Model 2, Table 3). Similarly, adjustment for pubertal development (Tanner Stage) did not make any difference to the size of these associations and was not included in final models owing to low response rates. ${ }^{21}$ Adjustment for maternal education and prepregnancy BMI attenuated some associations, however, this appeared important only for the relationship between DP scores at 13 years and FMI at 15 years (Model 3, Table 3). Both plausible dietary reporting and overreporting was associated with a lower FMI $z$-score compared with dietary underreporting.

The longitudinal analyses modelling change in DP and FMI $z$-scores (6772 individuals) adjusting for age, sex and dietary misreporting indicated that on average, a 1 s.d. unit increase in DP $z$-score between dietary assessments was associated with an increase in $\mathrm{FMI} z$-score of 0.04 s.d. units $(95 \% \mathrm{Cl}, 0.01-0.07)$. For each increasing year of age, the change in FMI $z$-score associated with a 1 s.d. increase in DP $z$-score reduced by $0.003(95 \% \mathrm{Cl}$, -0.005 to $0.0001, P$-value for interaction $=0.06$ ).

With each increase in quintile for the DP $z$-score between dietary assessments there was an increasing trend in FMI $z$-score ( $P$ for trend $=0.01$ ) (Figure 2). Respondents in the highest DP quintile had a FMI $z$-score that was on average, 0.13 s.d. units $(95 \% \mathrm{Cl}, 0.03-0.22)$ higher than those in the lowest quintile. For each increasing year of age, the change in FMI $z$-score associated with a one quintile increase in DP $z$-score attenuated by 0.002 s.d. units $(95 \% \mathrm{Cl}-0.004$ to $0.0004, P$-value for interaction $=0.10$ ).

Table 1. Mean age, BMI and body composition at 7-, 11-, 13- and 15-year follow-ups

\begin{tabular}{|c|c|c|c|c|c|c|c|c|}
\hline & \multicolumn{2}{|c|}{7 years } & \multicolumn{2}{|c|}{11 years } & \multicolumn{2}{|c|}{13 years } & \multicolumn{2}{|c|}{15 years } \\
\hline & Boys & Girls & Boys & Girls & Boys & Girls & Boys & Girls \\
\hline Age (years), $N$ & 4174 & 4050 & 3513 & 3629 & 3014 & 3120 & 2596 & 2905 \\
\hline Mean (s.d.) & $7.5(0.3)$ & $7.5(0.3)$ & $11.7(0.2)$ & $11.8(0.2)$ & $13.8(0.2)$ & $13.8(0.2)$ & $15.5(0.3)$ & $15.5(0.4)$ \\
\hline Range & $6.8-9.4$ & $7.0-9.4$ & $10.7-13.5$ & $10.4-13.6$ & $12.5-15.1$ & $12.6-15.2$ & $14.5-17.7$ & $14.3-17.5$ \\
\hline Fat mass $(\mathrm{kg}), N$ & & & 3438 & 3551 & 2956 & 3067 & 2454 & 2691 \\
\hline Mean (s.d.) & & & $10.4(6.6)$ & $13.1(6.8)$ & $11.1(7.7)$ & $16.4(7.6)$ & $11.4(8.5)$ & $19.0(8.4)$ \\
\hline Range & & & $1.5-47.3$ & $2.1-46.0$ & $1.9-55.5$ & $3.4-62.0$ & $2.1-61.3$ & $3.1-65.8$ \\
\hline Height $(\mathrm{cm}), N$ & 4174 & 4050 & 3496 & 3605 & 3010 & 3115 & 2572 & 2853 \\
\hline Mean (s.d.) & $126(6)$ & $125(6)$ & $150(7)$ & $151(7)$ & $165(9)$ & $162(6)$ & $174(8)$ & $165(6)$ \\
\hline Range & 104-153 & 103-157 & $126-179$ & $122-177$ & $132-196$ & $135-184$ & $145-200$ & $139-190$ \\
\hline$F M I^{\mathrm{a}}, N$ & & & 3432 & 3546 & 2955 & 3067 & 2454 & 2689 \\
\hline Mean (s.d.) & & & $1.2(0.7)$ & $2.3(1.1)$ & $4.4(3.0)$ & $3.6(1.6)$ & $3.0(2.2)$ & $5.8(2.5)$ \\
\hline Range & & & $0.2-5.1$ & $0.6-8.0$ & $0.9-19.3$ & $0.8-12.3$ & $0.6-16.6$ & $1.3-18.6$ \\
\hline Weight (kg), N & 4164 & 4047 & 3500 & 3604 & 3010 & 3107 & 2568 & 2846 \\
\hline Mean (s.d.) & $25.9(4.5)$ & $26(5)$ & $42.7(9.8)$ & $44.7(10.4)$ & $54.7(11.9)$ & $54.5(10.8)$ & $64.2(12.3)$ & $59.2(11.2)$ \\
\hline Range & $15.6-50.8$ & $14.0-67.2$ & $23.2-101.6$ & $22.6-105.4$ & $26.6-23.8$ & $27.4-125.4$ & $32.3-139.0$ & $37-122.8$ \\
\hline$B M I\left(\mathrm{~kg} \mathrm{~m}^{-2}\right), N$ & 4163 & 4047 & 3496 & 3600 & 3010 & 3107 & 2568 & 2846 \\
\hline Mean (s.d.) & $16.1(1.9)$ & $16.4(2.2)$ & $18.8(3.3)$ & $19.4(3.6)$ & $20.0(3.4)$ & 20.7 (3.6) & $21.0(3.4)$ & 21.9 (3.9) \\
\hline Range & $11.8-28.4$ & $10.8-34.9$ & $12.8-37.9$ & $12.4-36.8$ & $13.8-36.5$ & $13.5-44.2$ & $14.2-39.2$ & $14.1-63.6$ \\
\hline$\%$ Overweight or obese $\mathrm{e}^{\mathrm{b}}$ & 12.1 & 17.5 & 21.6 & 24.2 & 18.7 & 21.0 & 17.2 & 19.7 \\
\hline
\end{tabular}

Abbreviations: BMI, body mass index; FMI, fat mass index. ${ }^{a}$ Fat mass index calculated as fat mass (kg) divided by height ( $\mathrm{m}$ ) raised to an optimum power to remove the relationship between FMI and height, which varied by year and gender (FMI is not comparable by subgroup). ${ }^{b}$ Overweight or obese defined according to IOTF definitions for BMI. 


\begin{tabular}{|c|c|c|c|c|c|c|}
\hline \multicolumn{7}{|l|}{ Quintiles of DP $z$-scores at 7 years } \\
\hline$n$ & 1144 & 1134 & 1138 & 1121 & 1099 & \\
\hline BMI (s.e.) & $18.6(1.0)$ & $18.6(1.0)$ & $18.6(1.0)$ & $18.7(1.0)$ & $19.0(1.0)$ & $0.01^{\mathrm{a}}$ \\
\hline$\%$ Overweight or obese ${ }^{b}$ & 19 & 22 & 20 & 22 & 27 & $<0.0001^{c}$ \\
\hline$\%$ in highest Quintile FMI & 17 & 18 & 18 & 19 & 24 & $<0.0001^{\mathrm{c}}$ \\
\hline \multicolumn{7}{|l|}{ Quintiles of $D P Z$-scores at 10 years } \\
\hline \multicolumn{7}{|l|}{13 years of age } \\
\hline$n$ & 1166 & 1161 & 1146 & 1074 & 1079 & \\
\hline BMI (s.e.) & $20.1(1.0)$ & $20.0(1.0)$ & $20.1(1.0)$ & $19.9(1.0)$ & $20.2(1.0)$ & $0.20^{\mathrm{a}}$ \\
\hline$\%$ Overweight or obese ${ }^{\mathrm{b}}$ & 20 & 19 & 21 & 19 & 20 & $0.79^{c}$ \\
\hline \multicolumn{7}{|l|}{ Quintiles of DP $Z$-scores at 13 years } \\
\hline \multicolumn{7}{|l|}{15 years of age } \\
\hline$n$ & 1003 & 953 & 958 & 948 & 891 & \\
\hline BMI (s.e.) & $21.2(1.0)$ & $21.1(1.0)$ & $21.1(1.0)$ & $21.1(1.0)$ & $21.0(1.0)$ & $0.75^{\mathrm{a}}$ \\
\hline$\%$ Overweight or obese ${ }^{b}$ & 19 & 17 & 19 & 19 & 19 & $0.65^{c}$ \\
\hline$n$ & 975 & 934 & 946 & 928 & 879 & $0.52^{\mathrm{a}}$ \\
\hline FMl, $z$-score (s.e.) & $-0.02(0.03)$ & $-0.02(0.03)$ & $0.04(0.03)$ & $-0.01(0.03)$ & $-0.04(0.03)$ & \\
\hline$\%$ in highest Quintile FMI & 19 & 18 & 22 & 20 & 20 & $0.49^{c}$ \\
\hline
\end{tabular}

Higher scores for the energy-dense, high-fat, low-fibre DP were positively associated with greater odds of excess adiposity. For each 1 s.d. unit increase in DP $z$-score between dietary assessments, the odds increased by $13 \%$ (OR 95\% Cl, 1-27\%). There was little evidence of an interaction between DP $z$-score and age of dietary assessment $(P=0.20)$. Quintile analyses indicated an increasing trend between DP $z$-scores and the odds of excess adiposity; with each increase in DP quintile between dietary assessments, there was a $5 \%$ increase in odds (OR 95\% Cl, 3-8\%). Compared with those respondents in the lowest quintile for the DP score, those in the highest quintile had a $22 \%$ higher odds of excess adiposity (OR $95 \% \mathrm{Cl}, 10-35 \%)$ (Figure 3). There was no interaction between DP score quintile and age at dietary assessment $(P=0.53)$.

In all longitudinal models, physical activity and maternal education were negative predictors and maternal prepregnancy BMI was a positive predictor of FMI and excess adiposity. However, including these covariates in the GEE models made little or no difference to the reported associations (not shown but available from author on request).

The DP $z$-score is calculated using a weighted linear combination of all food group intakes. For example, in a 13-year-old female, substituting two slices ( $50 \mathrm{~g}$ per day) of high-fibre bread with low-fibre bread, $50 \mathrm{~g}$ per day of high-fibre breakfast cereal with low-fibre breakfast cereal and replacing $60 \mathrm{~g}$ per day vegetables with fried potato while keeping all other food intake constant, would result in a 1 s.d. unit increase in DP $z$-score.

\section{DISCUSSION}

This longitudinal study of UK children and adolescents followed from 7 to 15 years of age confirms that an energy-dense, high-fat, low-fibre DP is prospectively associated with greater levels of adiposity and excess adiposity. This implicates dietary energy density, fat and fibre intake as specific risk factors in the development of obesity during childhood and adolescence, and supports similar findings observed between 5 and 9 years of age in the same cohort. ${ }^{10}$

There were clear trends between higher scores for this type of DP and increased adiposity, across all ages. However, scores for the energy-dense, high-fat, low-fibre DP at 7 years of age showed a slightly stronger association with later adiposity than scores for the same pattern at 13 years of age. This finding did not support our hypothesis that the strength of association would increase with age, reflecting a decline in innate appetite control mechanisms. It may, however, reflect increasing levels of dietary measurement error with age of the study child. In this study, the prevalence of dietary misreporting increased with age and was most prevalent at 13 years of age. Respondents were likely to receive less assistance from a parent or guardian to complete their food diary at 13 years of age, and this may have led to a greater number of inaccuracies. Another contributing factor may be greater competing influences on an adolescent's adiposity at 13 years relative to younger ages, such as sedentary behaviours, smoking and alcohol use, which were not examined in this study.

There have been surprisingly few longitudinal studies on dietary intake and obesity in children or adolescents. Although some cross-sectional studies have examined individual foods or nutrients, for example, energy density, in relation to obesity in children, the findings have been inconsistent and frequently null. ${ }^{25}$ The current analysis and our previous analysis of ALSPAC participants between the ages of 5 and 9 years ${ }^{10}$ are the only studies, to our knowledge, that have examined RRR-derived DPs longitudinally in relation to adiposity in children and adolescents.

Two prospective studies have examined DPs derived using principal components analysis in relation to obesity in adolescents. A Norwegian study did not observe any prospective associations between four DPs and BMI in 9-10 years old over a 4-year follow-up. ${ }^{26}$ However, this study did not measure changes in scores for the exactly the same DPs, which may partly explain 
Table 3. Prospective associations between an energy-dense, high-fat, low-fibre DP (z-score) and fat mass index (z-score)

\begin{tabular}{|c|c|c|c|}
\hline Dietary pattern & FMI z-score & FMI z-score & FMI z-score \\
\hline Age & 11 years & 13 years & 15 years \\
\hline \multicolumn{4}{|l|}{7 years } \\
\hline \multicolumn{4}{|l|}{ Model 1} \\
\hline$n$ & 5501 & 4904 & 4245 \\
\hline Beta (s.e.) & $0.10(0.01)$ & $0.09(0.01)$ & $0.09(0.01)$ \\
\hline$P$-value & $<0.0001$ & $<0.0001$ & $<0.0001$ \\
\hline $95 \% \mathrm{Cl}$ & $0.07-0.12$ & $0.06-0.11$ & $0.06-0.12$ \\
\hline \multicolumn{4}{|l|}{ Model 2} \\
\hline$n$ & 4630 & 3983 & 3495 \\
\hline Beta (s.e.) & $0.09(0.01)$ & $0.09(0.02)$ & $0.09(0.02)$ \\
\hline$P$-value & $<0.0001$ & $<0.0001$ & $<0.0001$ \\
\hline $95 \% \mathrm{Cl}$ & $0.07-0.12$ & $0.06-0.11$ & $0.06-0.12$ \\
\hline \multicolumn{4}{|l|}{ Model 3} \\
\hline$n$ & 4002 & 3472 & 2626 \\
\hline Beta (s.e.) & $0.07(0.01)$ & $0.06(0.02)$ & $0.06(0.02)$ \\
\hline$P$-value & $<0.0001$ & 0.0001 & 0.0004 \\
\hline $95 \% \mathrm{Cl}$ & $0.05-0.10$ & $0.03-0.09$ & $0.03-0.10$ \\
\hline \multicolumn{4}{|l|}{10 years } \\
\hline \multicolumn{4}{|l|}{ Model 1} \\
\hline$n$ & 6356 & 5527 & 4736 \\
\hline Beta (s.e.) & $0.06(0.01)$ & $0.06(0.01)$ & $0.06(0.01)$ \\
\hline$P$-value & $<0.0001$ & $<0.0001$ & $<0.0001$ \\
\hline $95 \% \mathrm{Cl}$ & $0.05-0.08$ & $0.04-0.08$ & $0.04-0.09$ \\
\hline \multicolumn{4}{|l|}{ Model 2} \\
\hline$n$ & 5344 & 4560 & 3953 \\
\hline Beta (s.e.) & $0.06(0.01)$ & $0.07(0.01)$ & $0.06(0.01)$ \\
\hline$P$-value & $<0.0001$ & $<0.0001$ & $<0.0001$ \\
\hline $95 \% \mathrm{Cl}$ & $0.04-0.08$ & $0.04-0.09$ & $0.03-0.08$ \\
\hline \multicolumn{4}{|l|}{ Model 3} \\
\hline$n$ & 4571 & 3943 & 3425 \\
\hline Beta (s.e.) & $0.03(0.01)$ & $0.04(0.01)$ & $0.03(0.01)$ \\
\hline$P$-value & 0.005 & 0.002 & 0.023 \\
\hline $95 \% \mathrm{Cl}$ & $0.01-0.05$ & $0.01-0.06$ & $0.01-0.06$ \\
\hline \multicolumn{4}{|l|}{13 years } \\
\hline \multicolumn{4}{|l|}{ Model 1} \\
\hline$n$ & & & 4656 \\
\hline Beta (s.e.) & & & $0.03(0.01)$ \\
\hline$P$-value & & & 0.003 \\
\hline $95 \% \mathrm{Cl}$ & & & $0.01-0.05$ \\
\hline \multicolumn{4}{|l|}{ Model 2} \\
\hline$n$ & & & 3479 \\
\hline Beta (s.e.) & & & $0.03(0.01)$ \\
\hline$P$-value & & & 0.01 \\
\hline $95 \% \mathrm{Cl}$ & & & $0.01-0.05$ \\
\hline \multicolumn{4}{|l|}{ Model 3} \\
\hline$n$ & & & 3005 \\
\hline Beta (s.e.) & & & $0.01(0.01)$ \\
\hline$P$-value & & & 0.348 \\
\hline $95 \% \mathrm{Cl}$ & & & -0.01 to 0.03 \\
\hline
\end{tabular}

Abbreviations: $\mathrm{Cl}$, confidence interval; $\mathrm{FMl}$, fat mass index. Model 1: linear regression adjusted for age at dietary assessment, dietary misreporting and sex Model 2: as model 1, plus adjustment for total physical activity at 11 years. Model 3: as model 2, plus adjustment for maternal education and maternal prepregnancy BMI.

the lack of associations. In the US Project EAT study, there were no consistent prospective associations observed between 'vegetable', 'fruit', 'starchy food' or 'sweet and salty snack food' patterns and overweight or obesity according to BMI 5 years later. ${ }^{27} \mathrm{BMI}$ was

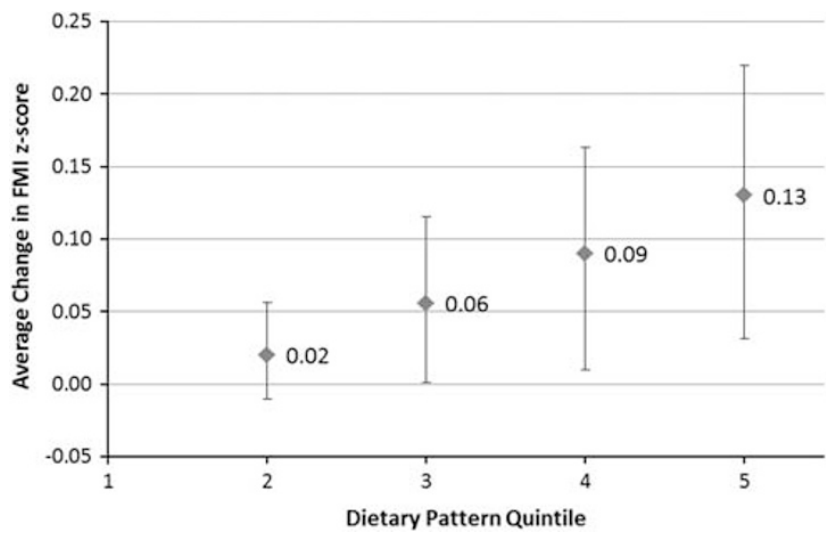

Figure 2. Average change in FMI $z$-score associated with change in quintile of the energy-dense, high-fat, low-fibre DP z-score. Average change in FMI $z$-score (beta coefficient) associated with an increase in DP quintile between dietary assessments, relative to 1st quintile. Adjusted for age at dietary assessment, age at outcome, dietary misreporting and gender. Error bars are $95 \% \mathrm{Cl}$ for beta coefficient. $P$-value for trend $=0.01$.

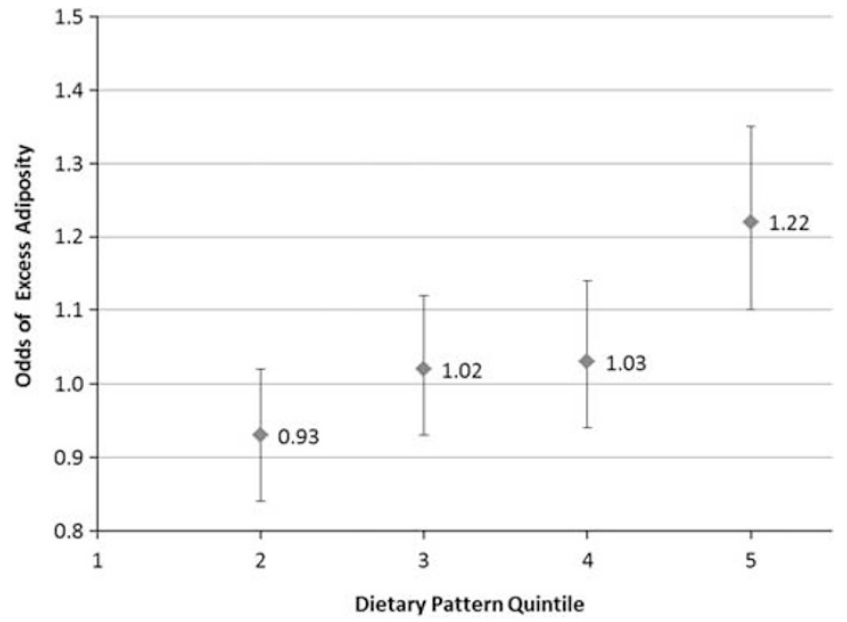

Figure 3. Odds of excess adiposity according to change in quintile of the energy-dense, high-fat, low-fibre DP $z$-score. Odds of excess adiposity associated with an increase in DP quintile between dietary assessments, relative to 1 st quintile. Adjusted for age at dietary assessment, age at outcome, dietary misreporting and gender. Error bars are $95 \% \mathrm{Cl}$ for odds ratio. $P$-value for trend $<0.001$

based on self-reported height and weight, and DP scores were measured once only (at baseline).

Like most cohort studies, the ALSPAC study population was slightly more affluent than the national average and included a smaller number of women from ethnic minorities. However, ALSPAC children showed almost identical growth standards to those for Britain as a whole. ${ }^{28}$ There was some attrition in the study population over time and there may be some bias among those attending follow-ups compared with those who did not. However, this analysis included over 6500 children and all available data were analysed rather than focussing on only those children who completed all follow-ups, reducing bias to some degree. Although we attempted to control for the other influences on children's diets, residual confounding cannot be ruled out.

This study has several major strengths. Data were collected approximately every 2 years, enabling a longitudinal analysis spanning 7-15 years of age. The use of 3-day food diaries provides detailed characterisation of dietary intake and avoids reliance on dietary recall. We attempted to adjust for dietary misreporting 
using an individualised method. The use of RRR enabled a specific DP to be examined; one hypothesised to have a relationship with adiposity on the basis of its energy density, fat and fibre content. RRR also has the advantage of considering all three of these dietary factors simultaneously in the form of a DP. The use of confirmatory RRR in this study provided repeated measures of exactly the same DP that could be modelled over time. Adiposity was assessed using direct measures of FM rather than BMI. Finally, the observed associations between the DP and adiposity in this study were independent of physical activity measured objectively with accelerometers, whereas most other studies have relied on physical activity questionnaires.

In conclusion, this analysis of UK children indicates that a DP that is high in energy density, high in fat and low in fibre is prospectively associated with greater adiposity in childhood and adolescence. This provides important information for interventions and policy in the area of obesity prevention in young people.

\section{CONFLICT OF INTEREST}

The authors declare no conflict of interest.

\section{ACKNOWLEDGEMENTS}

We are extremely grateful to all the families who took part in this study, the midwives for their help in recruiting them, and the whole ALSPAC team, which includes interviewers, computer and laboratory technicians, clerical workers, research scientists, volunteers, managers, receptionists and nurses. The current analysis was funded by a project grant from the World Cancer Research Fund (2008/31) and programme grant from the Medical Research Council (U105960389 Nutrition and Health). The Medical Research Council (Grant ref: 74882),the Wellcome Trust (Grant ref: 092731) and the University of Bristol provide core support for ALSPAC. LDH is funded by a Medical Research Council Population Health Scientist Fellowship. This publication is the work of the authors and GLA and SJ will serve as guarantors for the contents of this paper.

\section{REFERENCES}

1 Prentice AM, Poppitt SD. Importance of energy density and macronutrients in the regulation of energy intake. Int J Obes Relat Metab Disord 1996; 20 Suppl 2.

2 Cecil JE, Palmer CNA, Wrieden W, Murrie I, Bolton-Smith C, Watt P et al. Energy intakes of children after preloads: adjustment, not compensation. Am J Clin Nutr 2005; 82: 302-308.

3 Prentice AM, Jebb SA. Fast foods, energy density and obesity: a possible mechanistic link. Obesity Rev 2003; 4: 187-194.

4 Westerterp KR. Diet induced thermogenesis. Nutr Metab 2004; 1: 5.

5 Rolls BJ. The role of energy density in the overconsumption of fat. J Nutr 2000; 130: 268S-271S

$6 \mathrm{Du} \mathrm{H}$, Feskens EJM. Dietary determinants of obesity. Acta Cardiologica 2010; 65: 377-386.

7 Abete I, Astrup A, Martínez JA, Thorsdottir I, Zulet MA. Obesity and the metabolic syndrome: role of different dietary macronutrient distribution patterns and specific nutritional components on weight loss and maintenance. Nutrition Rev 2010; 68: 214-231.

8 World Health Organisation (WHO)and Food and Agriculture Organisation (FAO) of the United Nations. Diet, nutrition and the prevention of chronic diseases. Report of a joint WHO/FAO Expert Consultation. WHO Technical Report Series No. 916. WHO: Geneva, 2003
9 Hu FB. Dietary pattern analysis: a new direction in nutritional epidemiology. Curr Opin Lipidol 2002; 13: 3-9.

10 Johnson L, Mander AP, Jones LR, Emmett PM, Jebb SA. Energy-dense, low-fiber, high-fat dietary pattern is associated with increased fatness in childhood. Am J Clin Nutr 2008; 87: 846-854.

11 Golding J, Pembrey M, Jones J, Team TAS. ALSPAC—The Avon Longitudinal Study of Parents and Children. Paediatr Perinat Epidemiol 2001; 15: 74-87.

12 Rennie KL, Coward A, Jebb SA. Estimating under-reporting of energy intake in dietary surveys using an individualised method. Br J Nutr 2007; 97: 1169-1176.

13 Torun B. Energy requirements of children and adolescents. Public Health Nutr 2005; 8: 968-993.

14 Black AE, Cole TJ. Within- and between-subject variation in energy expenditure measured by the doubly-labelled water technique: implications for validating reported dietary energy intake. Euro J Clin Nutr 2000; 54: 386-394.

15 Hoffmann K, Schulze MB, Schienkiewitz A, Nothlings U, Boeing H. Application of a new statistical method to derive dietary patterns in nutritional epidemiology. Am J Epidemiol 2004; 159: 935-944.

16 SAS. SAS for Windows. 9.1.3 edn. SAS Institute Incorporated: Cary, NC, USA, 2002-2003.

17 Johnson L, Wilks DC, Lindroos AK, Jebb SA. Reflections from a systematic review of dietary energy density and weight gain: is the inclusion of drinks valid? Obesity Rev 2009; 10: 681-692.

18 Imamura F, Lichtenstein AH, Dallal GE, Meigs JB, Jacques PF. Generalizability of dietary patterns associated with incidence of type 2 diabetes mellitus. Am J Clin Nutr 2009; 90: 1075-1083.

19 Wells JCK, Cole TJ, Team TAS. Adjustment of fat free mass and fat mass for height in children aged 8 y. Int J Obesity 2002; 26: 947-952.

20 Tanner JM. Normal growth and techniques of growth assessment. Clin Endocrinol Metabol 1986; 15: 411-451.

21 Monteilh C, Kieszak S, Flanders WD, Maisonet M, Rubin C, Holmes AK et al. Timing of maturation and predictors of Tanner stage transitions in boys enrolled in a contemporary British cohort. Paediatr Perinat Epidemiol 2011; 25: 75-87.

22 Mattocks C, Ness A, Leary S, Tilling K, Blair SN, Shield J et al. Use of accelerometers in a large field-based study of children: protocols, design issues, and effects on precision. J Physic Activ Health 2008; 5: S98-S111.

23 Toschke AM, Martin RM, von Kries R, Wells J, Davey Smith G, Ness AR. Infant feeding method and obesity: body mass index and dual-energy X-ray absorptiometry measurements at 9-10 y of age from the Avon Longitudinal Study of Parents and Children (ALSPAC). Am J Clin Nutr 2007; 85: 1578-1585.

24 Stata. Stata/SE 11.2 for Windows. 11.2 edn. StataCorp LP: College Station, 2011.

25 Rodriguez G, Moreno LA. Is dietary intake able to explain differences in body fatness in children and adolescents? Nutr Metabol Cardiovasc Dis 2006; 16: 294-301.

26 Oellingrath IM, Svendsen MV, Brantsæter AL. Tracking of eating patterns and overweight-a follow-up study of Norwegian schoolchildren from middle childhood to early adolescence. Nutr J 2011; 10: 106.

27 Cutler GJ, Flood A, Hannan PJ, Slavin JL, Neumark-Sztainer D. Association between major patterns of dietary intake and weight status in adolescents. Br J Nutr 2011; 108: $349-356$.

28 Department of Social Medicine, University of Bristol. Avon Longitudinal Study of Parents and Children, 2008. http://www.bristol.ac.uk/alspac/sci-com/resource/ represent/(accessed 20 November 2011).

(c) This work is licensed under the Creative Commons AttributionOMERIGHISRESERVED NonCommercial-No Derivative Works 3.0 Unported License. To view a copy of this license, visit http://creativecommons.org/licenses/by-nc-nd/3.0/

Supplementary Information accompanies the paper on International Journal of Obesity website (http://www.nature.com/ijo) 